\title{
Comparisons of health status scores with MRC grades in COPD: implications for the GOLD 2011 classification
}

\author{
Paul W. Jones ${ }^{1}$, Lukasz Adamek², Gilbert Nadeau² and Norbert Banik ${ }^{3}$ \\ Affiliations: \\ 'Division of Clinical Science, St George's University of London, London, and \\ ${ }^{2}$ Respiratory Centre of Excellence, GlaxoSmithKline, Uxbridge, UK. \\ ${ }^{3}$ Biostatistics and Epidemiology, GlaxoSmithKline, Munich, Germany.
}

\section{Correspondence:}

P.W. Jones, Division of Clinical Science, St George's University of London, Cranmer Terrace, London, SW17 ORE, UK.

E-mail: pjonesdasghms.ac.uk

ABSTRACT The 2011 Global Initiative for Chronic Obstructive Lung Disease (GOLD) strategy document recommends assessment of chronic obstructive pulmonary disease (COPD) using symptoms and future exacerbation risk, employing two score cut-points: COPD Assessment Test (CAT) score $\geqslant 10$ or modified Medical Research Council dyspnoea scale (mMRC) grade $\geqslant 2$. To explore the equivalence of these two symptom cut-points, the relationship between the CAT and the mMRC and St George's Respiratory Questionnaire (SGRQ), the Short-form Health Survey and the Functional Assessment of Chronic Illness Therapy Fatigue scores were retrospectively analysed using a primary care dataset.

Data from 1817 patients (mean \pm SD forced expiratory volume in $1 \mathrm{~s} 1.6 \pm 0.6 \mathrm{~L}$ ) showed a significant association between mMRC grades and all health status scores (ANOVA $p<0.0001$ ). mMRC grade 1 was associated with significant levels of health status impairment (SGRQ 39.4 \pm 15.5 and CAT 15.7 \pm 7.0 ); even patients with mMRC grade 0 had modestly elevated scores (SGRQ 28.5 \pm 15.1 and CAT 11.7 \pm 6.8 ). An mMRC grading $\geqslant 2$ categorised $57.2 \%$ patients with low symptom (groups A and C) versus $17.2 \%$ with the CAT. Using the mMRC cut-point $(\geqslant 1)$ resulted in similar GOLD group categorisations as the CAT $(18.9 \%)$.

The mMRC showed a clear relationship with health status scores; even low mMRC grades were associated with health status impairment. Cut-points of mMRC grade $\geqslant 1$ and CAT score $\geqslant 10$ were approximately equivalent in determining low-symptom patients. The GOLD assessment framework may require refinement.

@ERSpublications

Low mMRC grades were linked with significantly impaired health; the GOLD COPD assessment framework may need refinement http://ow.ly/mAuis

Received: Aug 092012 | Accepted after revision: Nov 302012 | First published online: Dec 202012

Support statement: Funding for this study was provided by GlaxoSmithKline (GSK) (study number: 111749). Editorial support was funded by GSK.

Conflict of interest: Disclosures can be found alongside the online version of this article at www.erj.ersjournals.com

Copyright @ERS 2013 


\section{Introduction}

Chronic obstructive pulmonary disease (COPD) is a common chronic inflammatory disease, characterised by persistent airflow limitation, which is both preventable and treatable [1]. In individual patients, the overall severity of the disease is influenced by exacerbations and comorbidities [1]. Until recently, spirometry remained the standard method for grading COPD severity [2]. However, it is now recognised that, at the individual patient level, forced expiratory volume in $1 \mathrm{~s}$ (FEV1) is an unreliable marker of the severity of breathlessness, exercise limitation and health status impairment [3]. The 2011 Global Initiative for Chronic Obstructive Lung Disease (GOLD) strategy document now recommends that COPD management and treatment should consider both disease impact, determined by assessment of symptoms and activity limitation, and future risk of exacerbations, determined from airflow limitation or exacerbation history [1]. This combined assessment of COPD results in the grouping of patients into one of four categories: A: low risk, fewer symptoms; B: low risk, more symptoms; C: high risk, fewer symptoms; D: high risk, more symptoms.

GOLD recommends either the COPD Assessment Test (CAT) or the modified Medical Research Council dyspnoea scale (mMRC) for assessing symptoms. The CAT is a short, eight-item health status questionnaire developed to provide a simple tool for quantifying the symptoms and impacts of COPD [4]. It has been shown to distinguish between different severities of COPD and is highly correlated with the St George's Respiratory Questionnaire (SGRQ) [5]. The original Medical Research Council (MRC) dyspnoea scale was described by FLETCHER [6] in 1952 and was developed to help physicians establish clinical grades of breathlessness (five grades) for their patients with emphysema, based on their ability to perform physical activities. A modified version of this scale is used today, which has more simplified statements and refers to "people" instead of "men", but is based on a similar five stages of breathlessness due to physical exertion [7]. (Note that the original MRC grades ranged from 1 to 5; confusingly, the mMRC grades patients from 0 to 4.) BESTALL et al. [8] showed the MRC grade to be significantly associated with shuttle distance, SGRQ scores, Chronic Respiratory Questionnaire scores and The Nottingham Extended Activities of Daily Living scores. However, this study only evaluated patients with MRC grades 3-5 and not patients with milder disease (MRC grades 1 and 2). In the absence of other evidence, GOLD recommended a CAT score $\geqslant 10$ and mMRC score $\geqslant 2$ as equivalent symptom cut-points for categorising patients into low- or high-symptom groups.

The primary aim of this analysis was to test the equivalence, assumed by GOLD, between these two symptom cut-points, by examining the relationship between health status scores and mMRC grades in patients with COPD with a wide range of severity, using the Health-Related Quality of Life in COPD in Europe Study (HEED) database [9]. A secondary aim was to compare the demographic and clinical characteristics and health status scores of patients grouped using the new GOLD combined assessment framework.

\section{Methods}

The HEED study was a large, cross-sectional, observational study conducted in primary care, full details of which have been published previously [9]. Patients visiting their primary care physician for any reason and with a diagnosis of COPD (aged 40-80 years; post-bronchodilator FEV1/forced vital capacity (FVC) ratio of $<70 \%$; smoking pack history of $\geqslant 10$ pack-years) were invited to participate.

At a single study visit, patients completed the following health-related quality-of-life questionnaires: COPDspecific SGRQ(-C) [10]; the 12-item Short-form Health Survey (SF-12) [11]; the CAT [4]; the Functional Assessment of Chronic Illness Therapy (FACIT) fatigue scale [12]. Dyspnoea was assessed using the mMRC [7]. The number of patient-reported exacerbations in the previous 6 months was recorded. An exacerbation was defined as a worsening of symptoms that required oral corticosteroids and/or antibiotics and/or hospitalisation. Post-bronchodilator FEV1, FVC and FEV1/FVC ratio were also recorded; spirometry was either performed at the study visit or from documented evidence within the previous 6 months.

\section{Statistical analysis}

Sample size calculations have been described in an earlier publication [9].

Descriptive statistics using Statistical Analysis Systems version 9.1.3 software (SAS Inc, Cary, NC, USA), were used to report mean health status scores in each of the mMRC grades. ANOVA was applied to test the association between mMRC grade and each health status score.

Descriptive statistics were used to describe the demographic and baseline characteristics of the patients within each GOLD assessment category. Data on exacerbations over the preceding 6 months were collected under three headings: 1) exacerbations treated with antibiotics; 2) exacerbations treated with oral corticosteroids (OCS); and 3) exacerbations requiring hospitalisation. Since the total number of exacerbations was not collected in an individual, the sum of all three exacerbation categories was used and annualised. Using this approach, patients with no exacerbations in the preceding 6 months were 
classified into GOLD categories A and B. Patients with $\geqslant 1$ exacerbation in the preceding 6 months had an extrapolated exacerbation rate of $\geqslant 2$, so were categorised into groups $\mathrm{C}$ or $\mathrm{D}$.

A concordance analysis was applied to quantify the association between the frequencies of patients categorised into GOLD groups A to D using symptom cut-points CAT $\geqslant 10$ versus $\mathrm{mMRC} \geqslant 2$ and CAT $\geqslant 10$ versus $\mathrm{mMRC} \geqslant 1$, respectively. A weighted $\kappa$-coefficient was calculated (a value of 1 indicates a perfect agreement).

\section{Results}

\section{Study population}

The demographic and clinical characteristics of the reported population have been described previously [10]. The mean age was 65 years and mean duration of COPD was 9 years. The mean FEV1 was 1.6 L (57\% predicted) and $>40 \%$ were current smokers. In the previous 6 months, just over half of all patients had had an exacerbation requiring antibiotics, $35 \%$ had an exacerbation requiring OCS and 10\% had been hospitalised due to an exacerbation.

\section{Clinical characteristics of patients by new GOLD classification}

The demographic and clinical characteristics of patients split by the new GOLD groups, classified by CAT $\geqslant 10$ or MRC $\geqslant 2$ cut-points are shown in table 1 . The mean age of patients was similar across GOLD groups, whether classified by CAT or mMRC and there was no apparent difference in mean number of comorbidities across GOLD groups by either classification. The highest level of COPD symptoms (cough, sputum and dyspnoea) and lowest level of FEV1 were observed in group D, regardless of classification method. A higher proportion of group A patients, as classified by the mMRC, reported respiratory symptoms compared to those placed in this category by the CAT; this was also true for patients in group B (symptoms of sputum and cough) and group C (all symptoms).

\section{Health status scores by new GOLD classification}

Patients in all four groups, as categorised by the $\mathrm{mMRC} \geqslant 2$ cut-point, had worse health status scores and more fatigue (encompassing tiredness, weakness and difficulty in conducting usual activities), compared with the equivalent group categorised by a CAT score $\geqslant 10$ (table 2). The differences in scores between these two categorisation methods groups exceeded the minimum clinically important difference (MCID) for each questionnaire [13, 14] (Quality Metric Inc., Lincoln, RI, USA; personal communication), with the exception of SF-12 physical component scores (PCS) in group D. For patients categorised by the mMRC as having low symptoms (groups A and C), SGRQ scores were much higher (by approximately three times the MCID) than those categorised by CAT.

\section{Relationship between health status scores and mMRC grade}

The results of the one-way ANOVA to test the association of health status scores with mMRC grade showed a significant relationship between mMRC grade and SGRQ, SF-12 PCS, CAT and FACIT scores $(\mathrm{p}<0.0001$ for all) (fig. 1). The differences in SGRQ and FACIT scores between mMRC grades $0-1,1-2,2-3$ and 3-4 all exceeded the MCIDs for these questionnaires $[13,14]$. The differences in SF-12 PCS score also exceeded its MCID between mMRC grades $0-1,1-2$ and 2-3, but not between grades 3-4. Notably, even patients with mMRC grade 0 ("only breathless with strenuous exercise") had modestly elevated CAT and SGRQ scores, and a mean SF-12 score of 44.5, which is below the normalised score of 50 expected of the general population $[11,15]$. For patients with mMRC grade 1 ("breathless when hurrying on level or walking up a slight hill”) or above, significant levels of health status impairment were observed.

Proportion of patients in each GOLD group using CAT score $\geqslant 10$ and mMRC grade $\geqslant 2$ symptom cutpoints (GOLD recommendation)

The proportion of patients categorised into groups A to D differed according to the use of a GOLD symptom cut-point of mMRC grade $\geqslant 2$ (fig. $2 \mathrm{a}$ ) or CAT score $\geqslant 10$ (fig. $2 \mathrm{~b}$ ). The mMRC classified $57.2 \%$ patients as having low symptoms (groups A and C), compared with $17.2 \%$ with the CAT. Concordance analysis comparing the frequencies of patients categorised by these two variables, the mMRC grade $\geqslant 2$ and CAT score $\geqslant 10$ cut-points, resulted in a weighted $\kappa$-coefficient of 0.626 .

Proportion of patients in each GOLD group using CAT score $\geqslant 10$ and mMRC grade $\geqslant 1$ symptom cutpoints (amended criteria)

As an exploratory exercise, health status scores were investigated when patients were categorised using a mMRC cut-point of grade $\geqslant 1$. Using this cut-point, patients categorised by mMRC grade $\geqslant 1$ had similar mean health status and fatigue scores to those found with a CAT score cut-point of $\geqslant 10$ (table 2). 


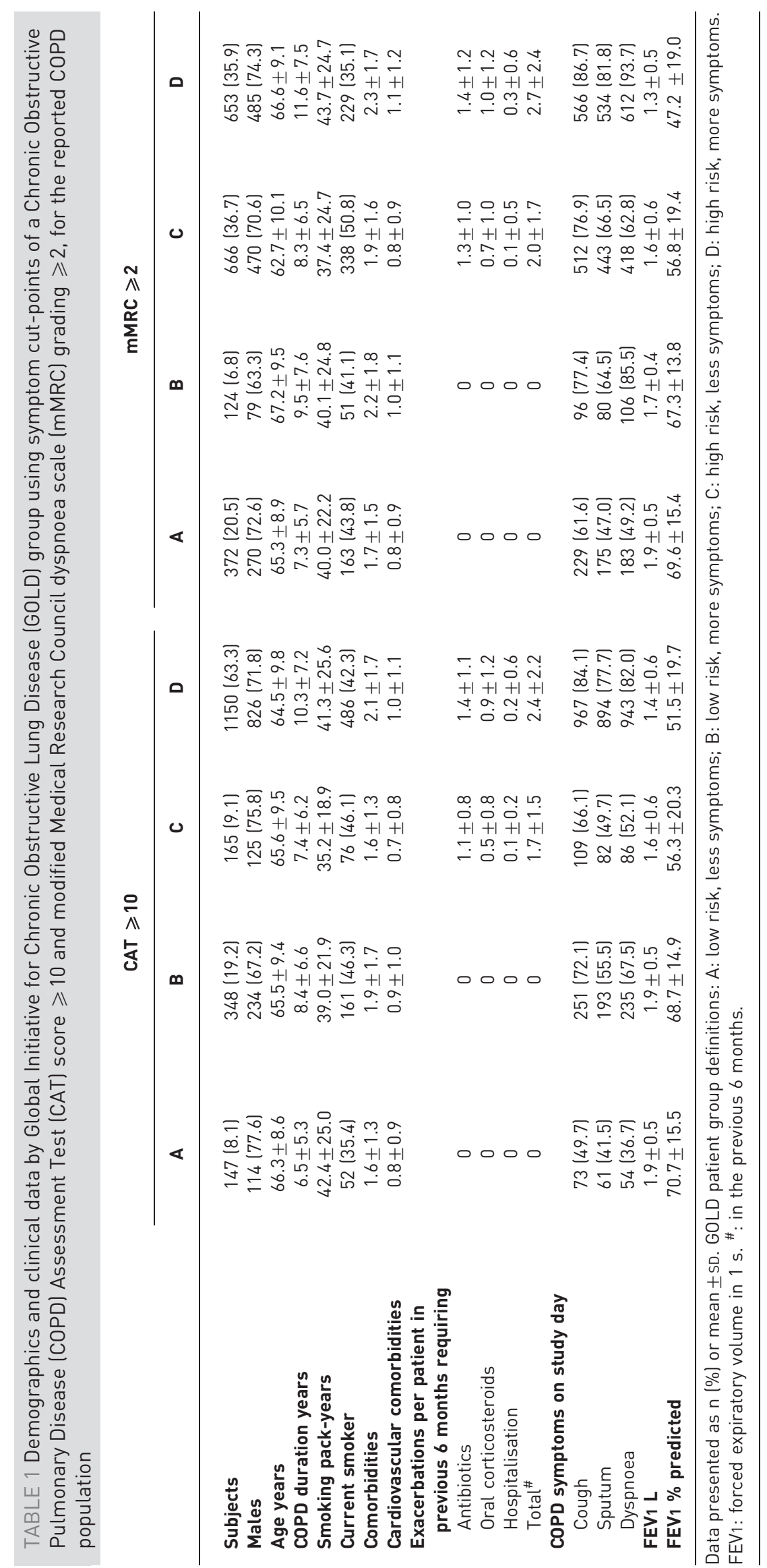


TABLE 2 Health status scores by Global Initiative for Chronic Obstructive Lung Disease (GOLD) group using symptom cutpoints of Chronic Obstructive Pulmonary Disease (COPD) Assessment Test (CAT) score $\geqslant 10$ and modified Medical Research Council dyspnoea scale (mMRC) grade $\geqslant 2$, and an exploratory cut-point using mMRC grade $\geqslant 1$, for the reported COPD population

Group

\begin{tabular}{lll}
\hline $\begin{array}{c}\text { SGRQ total } \\
\text { score }\end{array}$ & SF-12 PCS & FACIT $^{+}$
\end{tabular}

$\mathrm{mMRC} \geqslant 2$

\begin{tabular}{lll}
\hline $\begin{array}{c}\text { SGRQ total } \\
\text { score }\end{array}$ & SF-12 PCS & FACIT $^{+}$
\end{tabular}

mMRC $\geqslant 1$

\begin{tabular}{l}
\hline $\begin{array}{c}\text { SGRQ total } \\
\text { score }\end{array}$ \\
SF-12 PCS
\end{tabular}

$\begin{array}{lllllllllllll}\text { A } & 30.5(28.8-32.2) & 43.2(42.3-44.0) & 40.5(39.6-41.5) & 19.5(17.8-21.3) & 46.7(45.4-47.9) & 45.8(44.9-46.6) & 24.7(22.3-27.1) & 45.7(44.4-47.0) & 42.4(41.1-43.8)\end{array}$

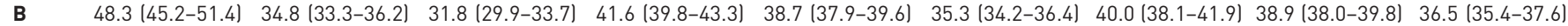

$\begin{array}{lllllllllllll}\text { C } & 38.6(37.3-39.9) & 40.3(39.7-41.0) & 38.0(37.3-38.7) & 24.2(22.5-26.0) & 45.1 & (43.9-46.3) & 44.5 & (43.4-45.6) & 31.8(29.5-34.0) & 43.4(42.3-44.6) & 40.4(39.1-41.7)\end{array}$

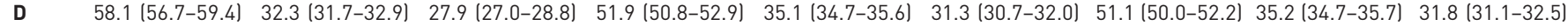

Data are presented as mean (95\% CI). SGRQ: St George's Respiratory Questionnaire; SF-12 PCS: 12-item Short-form Health Survey physical component score; FACIT: Functional Assessment of Chronic Illness Therapy fatigue scale. GOLD patient group definitions: A: low risk, less symptoms; B: low risk, more symptoms; C: high risk,

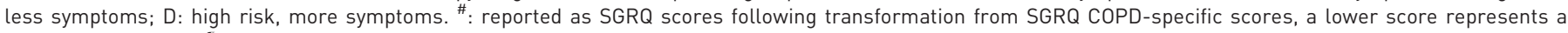
better quality of life; ${ }^{\natural}$ : a higher score represents a better quality of life; ${ }^{+}$: a higher score indicates less fatigue.
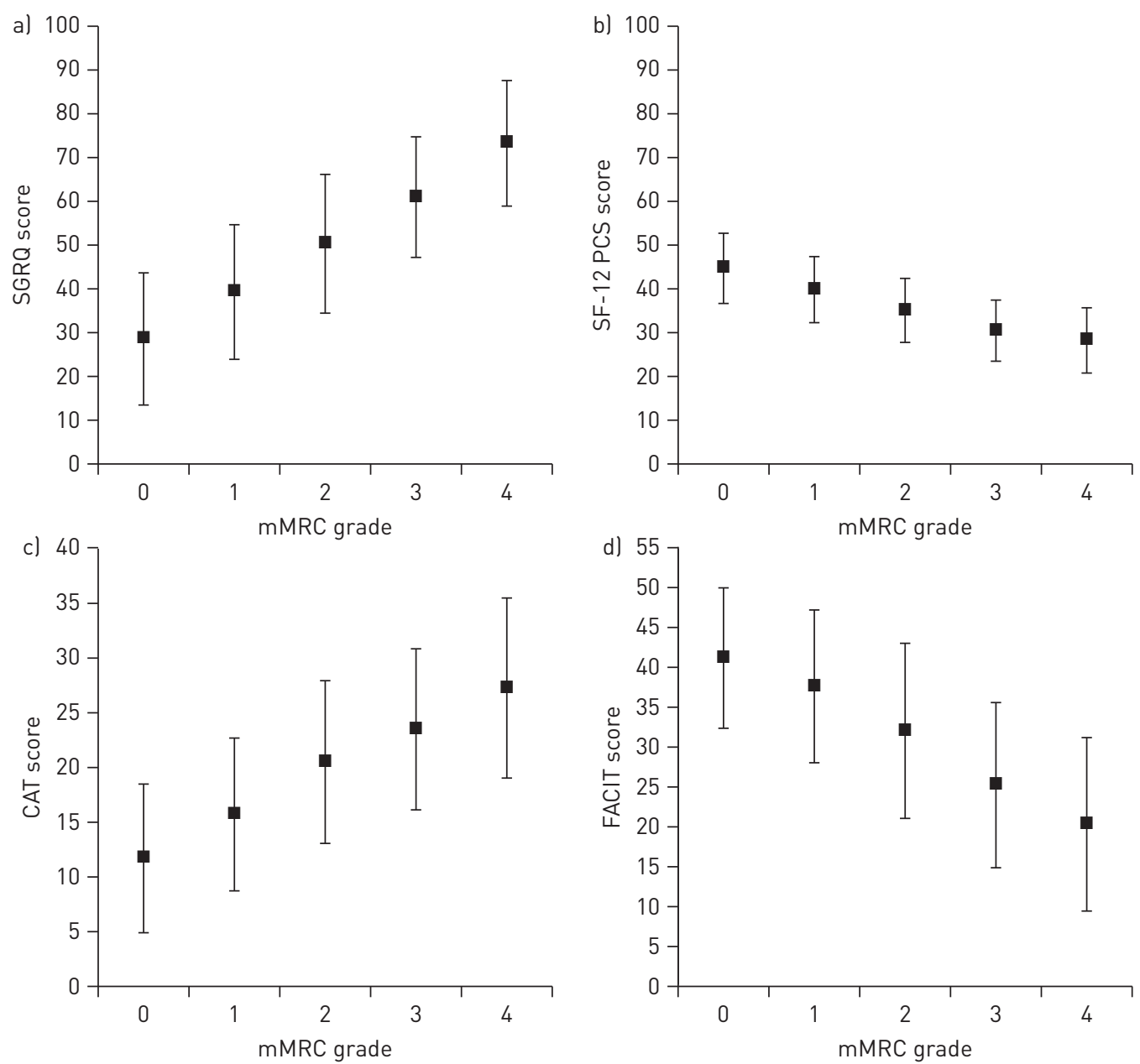

FIGURE 1 Health status scores by modified Medical Research Council dyspnoea scale (mMRC) grade for the reported chronic obstructive pulmonary disease (COPD) populations. a) St George's Respiratory Questionnaire (SGRQ); b) 12item Short-form Health Survey physical component score (SF-12 PCS); c) COPD Assessment Test (CAT); d) Functional Assessment of Chronic Illness Therapy (FACIT) score. Data are presented as mean \pm sD. One-way ANOVA of the association between mMRC grade and health status scores $\mathrm{p}<0.0001$ for all. 
a)

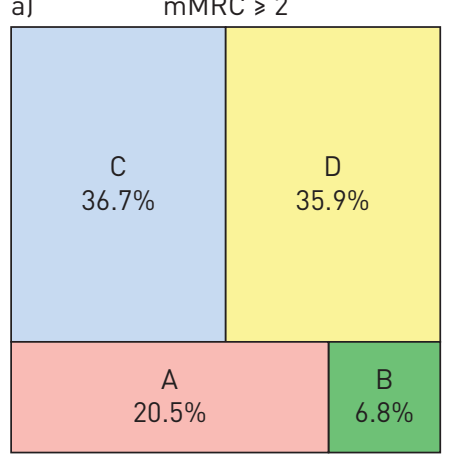

b)

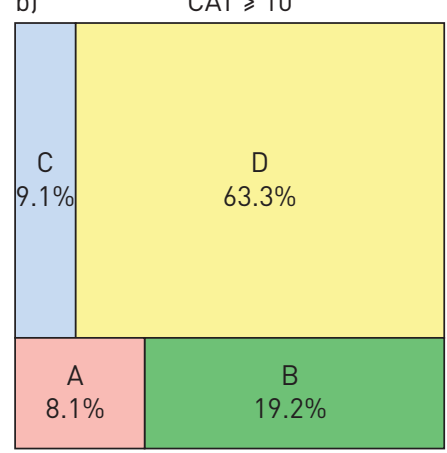

c)

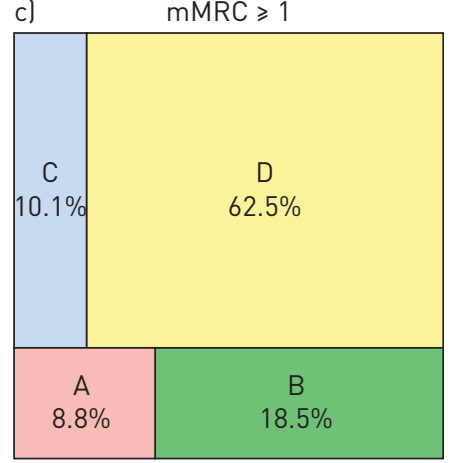

FIGURE 2 Proportion of patients in each Global Initiative for Chronic Obstructive Lung Disease (GOLD) group (A to D) using symptom cut-points. a) modified Medical Research Council dyspnoea scale (mMRC) grade $\geqslant 2$; b) Chronic Obstructive Pulmonary Disease (COPD) Assessment Test (CAT) score $\geqslant 10$; and c) mMRC grade $\geqslant 1$ for the reported COPD population.

Using this cut-point, the proportion of patients categorised in to groups A to D was similar to that with the CAT (fig. 2 c). mMRC grade $\geqslant 1$ classified $18.9 \%$ of patients as having low symptoms (groups A and C) compared with $17.2 \%$ with the CAT. Concordance analysis comparing the frequencies of patients categorised by these two variables, the mMRC grade $\geqslant 1$ and CAT score $\geqslant 10$ cut-points, resulted in a weighted $\kappa$ coefficient of 0.792 ; indicating that there was a higher degree of agreement between these variables and their respective cut-points than between the classification applying CAT $\geqslant 10$ and $\mathrm{mMRC} \geqslant 2$.

\section{Discussion}

The new GOLD assessment system incorporates symptoms and risk of exacerbations, in addition to an assessment of airflow limitation [1]. Two alternative symptomatic cut-points are recommended for use. In the absence of published evidence, mMRC grade $\geqslant 2$ and CAT score $\geqslant 10$ were thought to be equivalent. This analysis has shown that there is a clear relationship between mMRC grade and health status scores across the whole severity range of the mMRC scale, which supports and extends the findings of BESTALL et al. [8]. However, we also show that an mMRC score $\geqslant 2$ appears not to be equivalent to a CAT score $\geqslant 10$. Patients categorised as having low symptoms using this mMRC cut-point have significantly worse health status scores, and experience more fatigue, than patients categorised using the CAT with a cut-point of $\geqslant 10$. In this patient population (mean FEV1 57\% pred), mMRC grade $\geqslant 2$ categorised the majority of patients as having low symptoms (i.e. they were placed in GOLD groups A and C). The SGRQ scores in group $\mathrm{A}$ and $\mathrm{C}$ patients were much higher than those observed in COPD-diagnosed patients identified in a population study by FERRER et al. [16] and were very similar to the average SGRQ scores in COPD patients identified in another prevalence study [17]. By contrast, use of the CAT score $\geqslant 10$ cut-point placed a smaller proportion of patients in the low-symptom groups, although it should be noted that even these patients still had significant health status impairment as judged by SGRQ and SF-12.

The MRC dyspnoea scale was developed as a standard set of questions by experts in the field of chronic bronchitis and emphysema for measuring dyspnoea. FletCHER [6] reported that the use of the questions enabled different observers to get reasonably repeatable results and that there was general agreement between the answers to these questions and an objective measure of dyspnoea. It should be noted that most MRC grades contain two different activities, but neither the validity of such combinations nor the equivalence of the two combined activities has ever been tested. mMRC grade 1 describes a patient who reports being breathless when hurrying on the level or when walking up a slight hill. From a COPD perspective that might reflect a relatively less symptomatic patient than others with the condition; but from a broader perspective, outside the setting of COPD, being breathless when hurrying on the level indicates a significant level of symptoms that has an impact on normal daily activities. In this study, the mean SGRQ score in mMRC grade 1 patients was $39.4 \pm 15.5$, which lies within $0.5 \mathrm{SD}$ of the mean score in patients recruited to recent clinical trials $[18,19]$. Patients with mMRC grade 1 are clearly symptomatic, as judged by their health status scores.

The CAT was developed in a very different way from the mMRC; it focuses on all aspects of COPD and was developed following a rigorous selection of the items based on interviews and focus groups with COPD patients, supported by interviews with community physicians and pulmonologists [4]. The primary objective was to create a questionnaire made up of the smallest number of items that formed a unidimensional instrument with reliable measurement properties. Its testing was robust at each stage of 
development. The CAT correlates very well with the SGRQ [5] and a Bland-Altman plot shows that the two instruments perform in a very similar way across the scaling range of both instruments [20]. A CAT score $\geqslant 10$ has been shown to have a significant impact on the daily lives of patients with COPD [20]. Patients with a CAT score of $\geqslant 10$ are likely to be breathless on most days, get exhausted easily and take a long time to do housework. Higher CAT scores are associated with greater disease impacts.

An unexpected finding was that $15 \%$ of the patients in group B (i.e. with mMRC grade $\geqslant 2$ ) recorded no breathlessness using a simple self-reported measure of respiratory symptoms (table 1). This observation lends support to the use of standardised instruments such as the mMRC and CAT for assessing symptomatic effects of COPD, rather relying on the patient's response to global questions about symptoms and their severity.

The intention of the new GOLD assessment framework is for it to be used in clinical settings, so physicians need to be assured that the suggested cut-points are correct and meaningful. Misclassification of patients could potentially affect their future management and treatment. This analysis provides the first evidencebased approach to choosing the correct cut-points. Following our observation of a lack of concordance between CAT score $\geqslant 10$ and mMRC grade $\geqslant 2$, we carried out an exploratory analysis using different mMRC cut-points for the GOLD symptomatic classification. This was based on our observation that the mean CAT score in mMRC grade 0 patients was $11.7 \pm 6.8$. The characteristics of patients categorised using a mMRC grade $\geqslant 1$ cut-point matched those categorised by CAT score $\geqslant 10$ much more closely than the GOLD mMRC grade $\geqslant 2$ cut-point. The distribution of the patients into the four GOLD categories by CAT score $\geqslant 10$ and $\mathrm{mMRC}$ grade $\geqslant 1$ was very similar. The association between the classifications using a CAT score $\geqslant 10$ and the mMRC is stronger (weighted $\kappa$-coefficient closer to 1 ) when applying a cut-off of grade 1 instead of grade 2 for the mMRC-based classification. This does not mean that exactly the same patients were placed into the different categories using CAT and mMRC grade $\geqslant 1$, but it shows that the characteristics of the patients so categorised was similar. The differences in classification of patients using CAT or mMRC grade $\geqslant 1$ would influence treatment in only a small proportion of patients. By contrast, differences in the classification of patients using CAT or mMRC grade $\geqslant 2$ show that, using this mMRC cutpoint, a sizeable proportion of patients with symptoms that have a significant impact upon their daily activity would not be prescribed maintenance treatment from which they would be likely to derive some benefit. These data suggest that the new GOLD assessment framework symptom cut-points may require some future modification with respect to the mMRC.

One of the aims of this analysis was to use a large COPD dataset to examine the characteristics of patients grouped using the new GOLD combined assessment framework in a typical clinical practice setting. Overall, there were no marked differences in demographic characteristics when patients were categorised using the mMRC or the CAT. The overall level of comorbidities and cardiovascular comorbidities were similar across GOLD groups using either classification, which agrees with the findings for numbers of comorbidities split by the GOLD spirometric staging system [9].

A limitation of this analysis is that the individual total numbers of exacerbations over 12 months were not directly available and were derived from the sum of the numbers of exacerbations treated with antibiotics and/or treated with oral corticosteroids and/or leading to hospitalisation, and therefore may have overestimated the true number of total exacerbations. Also, spirometry was not performed in a standardised way across centres but fulfilled local requirements at each site. This study was conducted in primary care in patients with confirmed COPD and therefore may not be applicable to the total COPD population and does not represent patients with undiagnosed disease. A further limitation was that this was a cross-sectional study and we cannot speculate about movement across category boundaries over time.

The new GOLD combined COPD assessment provides a much-needed framework for measuring the impact of COPD in terms of current symptoms and future exacerbation risk. This analysis showed a clear relationship between $\mathrm{mMRC}$ and other health status scores, and that even patients with low mMRC grades have perceptible health status impairment. However, the current mMRC cut-point of grade $\geqslant 2$ appears to overestimate the proportion of patients with low symptoms; these data may provide evidence for the future modification of the group cut-off points with respect to mMRC scores.

\section{Acknowledgements}

The authors would also like to acknowledge editorial support in the form of development of the manuscript first draft, assembling tables and figures, collating author comments, copy-editing, and referencing which was provided by Kate Hollingworth of Continuous Improvement Ltd (Kingsbridge, UK). 


\section{References}

1 Global Initiative for Chronic Obstructive Lung Disease (GOLD). Global Strategy for the Diagnosis, Management and Prevention of COPD. www.goldcopd.com/guidelines-global-strategy-for-diagnosis-management.html Date last accessed: July 2012. Date last updated: February 2013.

2 The Global Initiative for Chronic Obstructive Lung disease (GOLD). 2010 Update: GOLD Report, Global Strategy for the Diagnosis, Management and Prevention of COPD. www.goldcopd.com.

3 Agusti A, Calverley PMA, Celli B, et al. Characterisation of COPD heterogeneity in the ECLIPSE cohort. Respir Res 2010; 11: 122-135.

4 Jones PW, Harding G, Berry P, et al. Development and first validation of the COPD Assessment Test. Eur Respir J 2009; 34: 648-654.

5 Jones PW, Brusselle G, Dal Negro RW, et al. Properties of the COPD assessment test in a cross-sectional European study. Eur Respir J 2011; 38: 29-35.

6 Fletcher CM. The clinical diagnosis of pulmonary emphysema; an experimental study. Proc R Soc Med 1952; 45: 577-584.

7 Mahler DA, Wells CK. Evaluation of clinical methods for rating dyspnoea. Chest 1988; 93: 580-586.

8 Bestall JC, Paul EA, Garrod R, et al. Usefulness of the Medical Research Council (MRC) dyspnoea scale as a measure of disability in patients with chronic obstructive pulmonary disease. Thorax 1999; 54: 581-586.

9 Jones PW, Brusselle G, Dal Negro RW, et al. Health-related quality of life in patients by COPD severity within primary care in Europe. Respir Med 2011; 1: 57-66.

10 Meguro M, Barley EA, Spencer S, et al. Development and validation of an improved, COPD specific version of the St George Respiratory Questionnaire. Chest 2007; 132: 456-463.

11 Ware JE, Kosinski M, Keller SD. A 12-item short form health survey: construction of scales and preliminary tests of reliability and validity. Med Care 1996; 34: 220-233.

12 Webster K, Odom L, Peterman A, et al. The Functional Assessment of Chronic Illness Therapy (FACIT) measurement system: validation of version 4 of the core questionnaire. Qual Life Res 1999; 8: 604.

13 Jones PW. Interpreting thresholds for a clinically significant change in health status in asthma and COPD. Eur Respir J 2002; 19: 398-404.

14 Yost KJ, Eton DT. Combining distribution- and anchor-based approaches to determine minimally important differences. The FACIT experience. Eval Health Prof 2005; 28: 172-191.

15 Kontodimopoulos N, Pappa E, Niakas D, et al. Validity of SF-12 summary scores in a Greek general population. Health Qual Life Outcomes 2007; 5: 55.

16 Ferrer M, Villasante C, Alonso J, et al. Interpretation of quality of life scores from the St George's Respiratory Questionnaire. Eur Respir J. 2002; 19: 405-413.

17 Miravitlles M, Soriano JB, Garcia-Rio F, et al. Prevalence of COPD in Spain: impact of undiagnosed COPD on quality of life and daily life activities. Thorax 2009; 64: 863-868.

18 Tashkin DP, Celli B, Senn S, et al. A 4-year trial of tiotropium in chronic obstructive pulmonary disease. $N$ Engl $J$ Med 2008; 359: 1543-1554.

19 Chapman KR, Rennard SI, Dogra A, et al. Long-term safety and efficacy of indacaterol, a long-acting $\beta_{2}$-agonist, in subjects with COPD. Chest 2011; 140: 68-75.

20 Jones $\mathrm{P}$, Tabberer $\mathrm{M}$, Chen WH. Creating scenarios of the impact of COPD and their relationship to COPD Assessment Test $\left(\mathrm{CAT}^{\mathrm{TM}}\right)$ scores. BMC Pulm Med 2011; 11: 42. 